Supplement of

\title{
Can controlled drainage control agricultural nutrient emissions? Evidence from a BACI experiment combined with a dual isotope approach
}

Mette V. Carstensen ${ }^{1}$, Jane R. Poulsen ${ }^{1}$, Niels B. Ovesen ${ }^{1}$, Christen D. Børgesen ${ }^{2}$, Søren K. Hvid ${ }^{3}$, Brian Kronvang ${ }^{1}$

${ }^{1}$ Department of Bioscience, Aarhus University, Vejlsøvej 25, 8600 Silkeborg, Denmark

${ }^{2}$ Department of Agroecology, Blichers Allé 20, 8830 Tjele, Denmark

${ }^{3}$ SEGES, Agro Food Park 15, 8200 Aarhus N, Denmark

*Correspondence to Mette V. Carstensen (mvc@bios.au.dk)

10 pages, 3 tables, 14 figures 
TABLE S1. TEXTURE OF IP1, IP2, CP1, AND CP2 FOLLOWING USDA CLASSIFICATION. . .3

TABLE S2. CACO3, TOTAL C, TOTAL N, AND C:N FOR IP1, IP2, CP1, AND CP2.

*B.D.L. $=B E L O W$ DETECTION LIMIT . 4

TABLE S3. BACI TEST OF THE SPATIALLY MONITORED GROUNDWATER LEVELS IN THE PERIODS BEFORE (11/05/12-31/03/13), AFTER1 (12/10/13-03/11/14), AND AFTER2 (11/17/14-03/09/15)..........................

FIGURE S1. TN AT CP1 AND IP1 DURING Y0 (2012/13), Y1 (2013/14), AND Y2 (2014/15)...........................

FIGURE S2. $\mathrm{NH}_{4}{ }^{+}$-N AT CP1 AND IP1 DURING Y0 (2012/13), Y1 (2013/14), AND Y2 (2014/15)....................6

FIGURE S3. N2O-N AT CP1 AND IP1 DURING Y0 (2012/13), Y1 (2013/14), AND Y2 (2014/15)......................6

FIGURE S4. TP AT CP1 AND IP1 DURING Y0 (2012/13), Y1 (2013/14), AND Y2 (2014/15)............................6

FIGURE S5. PO ${ }_{4}^{3-}$ AT CP1 AND IP1 DURING Y0 (2012/13), Y1 (2013/14), AND Y2 (2014/15) .........................

FIGURE S6. $\mathrm{SO}_{4}{ }^{2-}$ AT CP1 AND IP1 DURING Y0 (2012/13), Y1 (2013/14), AND Y2 (2014/15).........................

FIGURE S7. DRAIN FLOW AND PRECIPITATION AT CP2 AND IP2 DURING Y0 (2012/13), Y1 (2013/14),

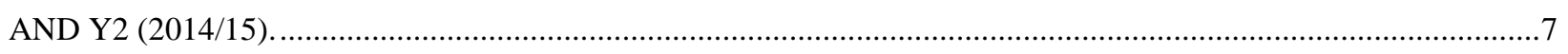

FIGURE S8. TN AT CP2 AND IP2 DURING Y0 (2012/13), Y1 (2013/14), AND Y2 (2014/15). ..........................

FIGURE S9. $\mathrm{NO}_{3}{ }^{-}-\mathrm{N}$ AT CP2 AND IP2 DURING Y0 (2012/13), Y1 (2013/14), AND Y2 (2014/15).......................

FIGURE S10. $\mathrm{NH}_{4}{ }^{+}$-N AT CP2 AND IP2 DURING Y0 (2012/13), Y1 (2013/14), AND Y2 (2014/15)....................

FIGURE S11. N 2 O-N AT CP2 AND IP2 DURING Y0 (2012/13), Y1 (2013/14), AND Y2 (2014/15).....................

FIGURE S12. TP AT CP2 AND IP2 DURING Y0 (2012/13), Y1 (2013/14), AND Y2 (2014/15) ..........................

FIGURE S13. PO ${ }_{4}^{3-}$ AT CP2 AND IP2 DURING Y0 (2012/13), Y1 (2013/14), AND Y2 (2014/15).......................

FIGURE S14. MEASURED RANGES OF $\Delta^{15} \mathrm{~N}$ AND $\Delta^{18}$ O OF NO ${ }^{-}-\mathrm{N}$ FROM IP1, IP2, CP1, AND CP2 IN Y1 (2013/14) AND Y2 (2014/15) PLOTTED WITH TYPICAL RANGES OF $\Delta{ }^{15} \mathrm{~N}$ AND $\Delta^{18} \mathrm{O}$ FROM NO${ }_{3}{ }^{-} \mathrm{N}$ SOURCES ADOPTED FROM KENDALL, ET AL. '. 


\begin{tabular}{|c|c|c|c|c|c|c|}
\hline \multirow[b]{3}{*}{ Plot } & \multirow[b]{3}{*}{ Horizon } & \multirow{3}{*}{$\begin{array}{l}\text { Clay } \\
(<2 \mu \mathrm{m}) \\
\mathrm{g} / 100 \mathrm{~g}\end{array}$} & \multirow{3}{*}{$\begin{array}{l}\text { Silt } \\
(2-50 \mu \mathrm{m}) \\
\mathrm{g} / 100 \mathrm{~g}\end{array}$} & \multirow{3}{*}{$\begin{array}{l}\text { Sand } \\
(50-2000 \mu \mathrm{m}) \\
\mathrm{g} / 100 \mathrm{~g}\end{array}$} & \multicolumn{2}{|l|}{ Humus } \\
\hline & & & & & & \\
\hline & & & & & $\mathrm{g} / 100 \mathrm{~g}$ & Soil type \\
\hline \multirow[t]{5}{*}{ IP1 } & Ap & 14.9 & 47.8 & 34.2 & 3.1 & Silt loam \\
\hline & Ap2 & 15.5 & 49.4 & 32.6 & 2.6 & Silt loam \\
\hline & Beg & 17.1 & 55.5 & 26.9 & 0.5 & Silt loam \\
\hline & Btg & 25.9 & 50.9 & 22.7 & 0.5 & Silt loam \\
\hline & $\mathrm{Lq}$ & 33.1 & 41.9 & 13.8 & 11.2 & Silty clay loam \\
\hline \multirow[t]{3}{*}{ IP2 } & Ap & 13.4 & 49.1 & 34.5 & 3.1 & Silt loam \\
\hline & Beg & 12.5 & 74.8 & 12.4 & 0.3 & Silt loam \\
\hline & Btg & 14.5 & 37.9 & 47.3 & 0.3 & Loam \\
\hline \multirow[t]{3}{*}{$\mathrm{CP} 1$} & Ap & 13.4 & 47.7 & 36.0 & 2.9 & Silt loam \\
\hline & Beg & 14.5 & 44.2 & 41.0 & 0.3 & Loam \\
\hline & $\mathrm{Cg}$ & 11.0 & 32.7 & 40.7 & 0.3 & Loam \\
\hline \multirow[t]{4}{*}{$\mathrm{CP} 2$} & Ap & 14.1 & 41.3 & 41.7 & 2.9 & Loam \\
\hline & $\mathrm{Be}$ & 14.9 & 45.4 & 39.2 & 0.5 & Loam \\
\hline & Btg & 17.1 & 42.6 & 32.6 & 0.3 & Silt loam \\
\hline & $\mathrm{Ccg}$ & 10.4 & 30.9 & 43.0 & 0.3 & Loam \\
\hline
\end{tabular}




\begin{tabular}{|c|c|c|c|c|c|}
\hline \multirow{3}{*}{ Plot } & \multirow{3}{*}{ Horizon } & \multirow{3}{*}{$\begin{array}{l}\mathrm{CaCO}_{3} \\
\mathrm{~g} / 100 \mathrm{~g}\end{array}$} & \multirow{3}{*}{$\begin{array}{l}\text { Total C } \\
\mathrm{g} / 100 \mathrm{~g}\end{array}$} & \multirow{3}{*}{$\begin{array}{l}\text { Total N } \\
\mathrm{g} / 100 \mathrm{~g}\end{array}$} & \multirow{3}{*}{$\begin{array}{l}\text { C:N } \\
\text { N/A }\end{array}$} \\
\hline & & & & & \\
\hline & & & & & \\
\hline \multirow[t]{5}{*}{ IP1 } & Ap & b.d.l. & 1.83 & 0.19 & 10 \\
\hline & Ap2 & b.d.l. & 1.50 & 0.16 & 9 \\
\hline & Beg & b.d.l. & 0.28 & 0.03 & 9 \\
\hline & Btg & b.d.l. & 0.31 & 0.03 & 10 \\
\hline & $\mathrm{Lq}$ & b.d.l. & 6.57 & 0.19 & 35 \\
\hline \multirow[t]{3}{*}{ IP2 } & Ap & b.d.l. & 1.84 & 0.17 & 11 \\
\hline & Beg & b.d.l. & 0.16 & 0.02 & 8 \\
\hline & Btg & b.d.l. & 0.16 & 0.01 & 16 \\
\hline \multirow[t]{3}{*}{$\mathrm{CP} 1$} & Ap & b.d.l. & 1.68 & 0.16 & 11 \\
\hline & Beg & b.d.l. & 0.20 & 0.02 & 10 \\
\hline & $\mathrm{Cg}$ & 15.43 & 2.02 & 0.01 & 202 \\
\hline \multirow[t]{4}{*}{$\mathrm{CP} 2$} & Ap & b.d.l. & 1.70 & 0.17 & 10 \\
\hline & $\mathrm{Be}$ & b.d.l. & 0.29 & 0.03 & 10 \\
\hline & Btg & 7.34 & 1.06 & 0.02 & 53 \\
\hline & $\mathrm{Ccg}$ & 15.51 & 2.03 & 0.01 & 203 \\
\hline
\end{tabular}


Table S3. BACI test of the spatially monitored groundwater levels in Y0 (2012/13), Y1 (2013/14), and Y2 (2014/15).

\begin{tabular}{|c|c|c|c|c|c|}
\hline Location at plot & Compared periods & $\mathrm{n}$ & $\begin{array}{l}\mathrm{BE} \\
(\mathrm{cm})\end{array}$ & t value & $\mathrm{p}$ \\
\hline \multirow[t]{2}{*}{ Center of plots } & $\mathrm{Y} 0 / \mathrm{Y} 1$ & 69 & -0.3 & 0.2 & 0.8 \\
\hline & $\mathrm{Y} 0 / \mathrm{Y} 2$ & 68 & -2.8 & -0.1 & 0.9 \\
\hline \multirow[t]{2}{*}{ Northeast of well } & $\mathrm{Y} 0 / \mathrm{Y} 1$ & 69 & -7.0 & 1.7 & 0.09 \\
\hline & $\mathrm{Y} 0 / \mathrm{Y} 2$ & 68 & -20.5 & 4.5 & $<0.001$ \\
\hline \multirow[t]{2}{*}{ Northwest of well } & Y0/Y1 & 69 & 5.3 & -1.7 & 0.09 \\
\hline & $\mathrm{Y} 0 / \mathrm{Y} 2$ & 68 & 5.6 & -2.9 & $<0.05$ \\
\hline \multirow{2}{*}{$\begin{array}{l}\text { Southwest of } \\
\text { well }\end{array}$} & Y0/Y1 & 69 & -2.6 & 0.8 & 0.4 \\
\hline & $\mathrm{Y} 0 / \mathrm{Y} 2$ & 68 & -2.8 & -0.4 & 0.7 \\
\hline \multirow[t]{2}{*}{ Closest to well } & $\mathrm{Y} 0 / \mathrm{Y} 1$ & 69 & $-6,3$ & 1,2 & 0.22 \\
\hline & $\mathrm{Y} 0 / \mathrm{Y} 2$ & 68 & $-18,4$ & 6,0 & $<0.001$ \\
\hline \multirow[t]{2}{*}{ East of the plot } & Y0/Y1 & 69 & -9.6 & 5,3 & $<0.001$ \\
\hline & $\mathrm{Y} 0 / \mathrm{Y} 2$ & 68 & -12.0 & 5,7 & $<0.001$ \\
\hline \multirow[t]{2}{*}{ South of the plot } & Y0/Y1 & 69 & -7.5 & 1,2 & 0.25 \\
\hline & Y0/Y2 & 68 & -11.4 & 2,8 & $<0.05$ \\
\hline
\end{tabular}
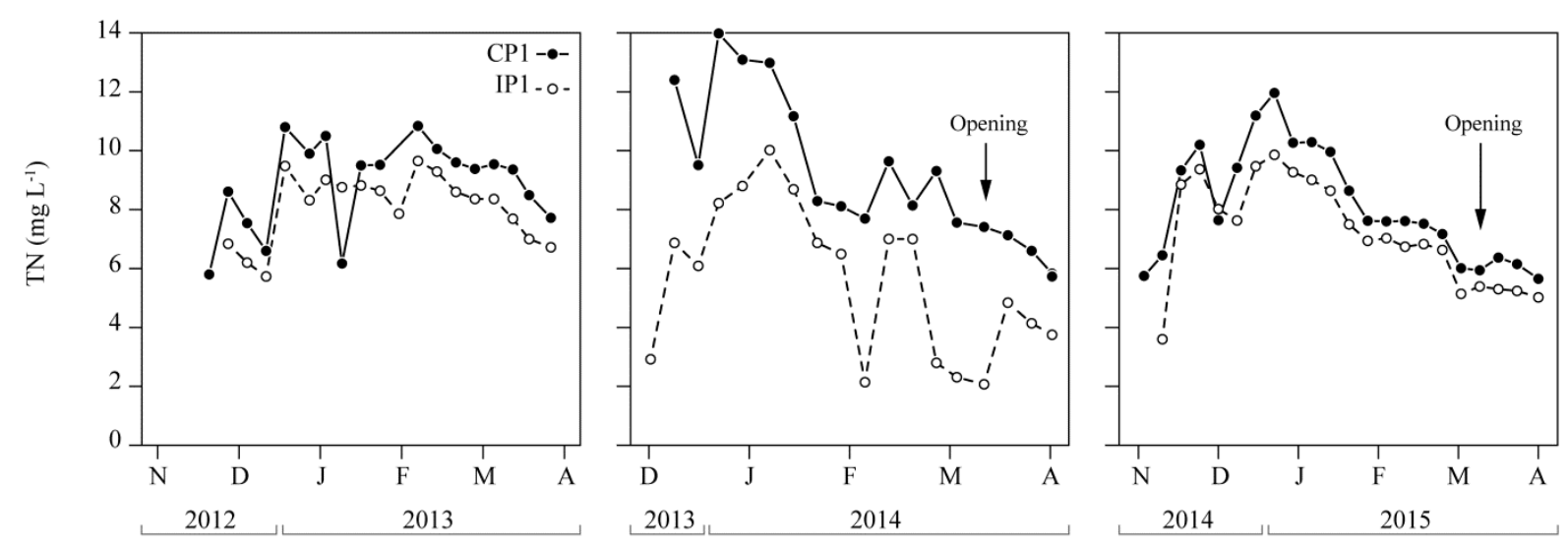

Figure S1. TN at CP1 and IP1 during Y0 (2012/13), Y1 (2013/14), and Y2 (2014/15). 

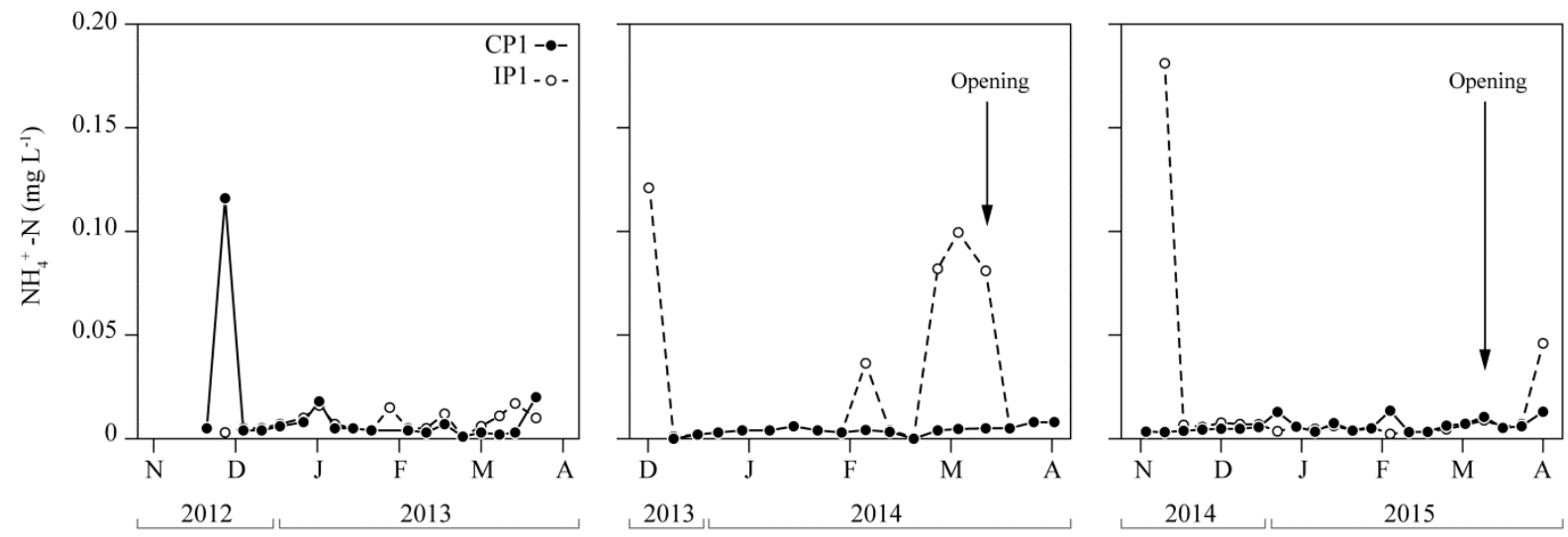

Figure S2. $\mathrm{NH}_{4}{ }^{+}$-N at CP1 and IP1 during Y0 (2012/13), Y1 (2013/14), and Y2 (2014/15).
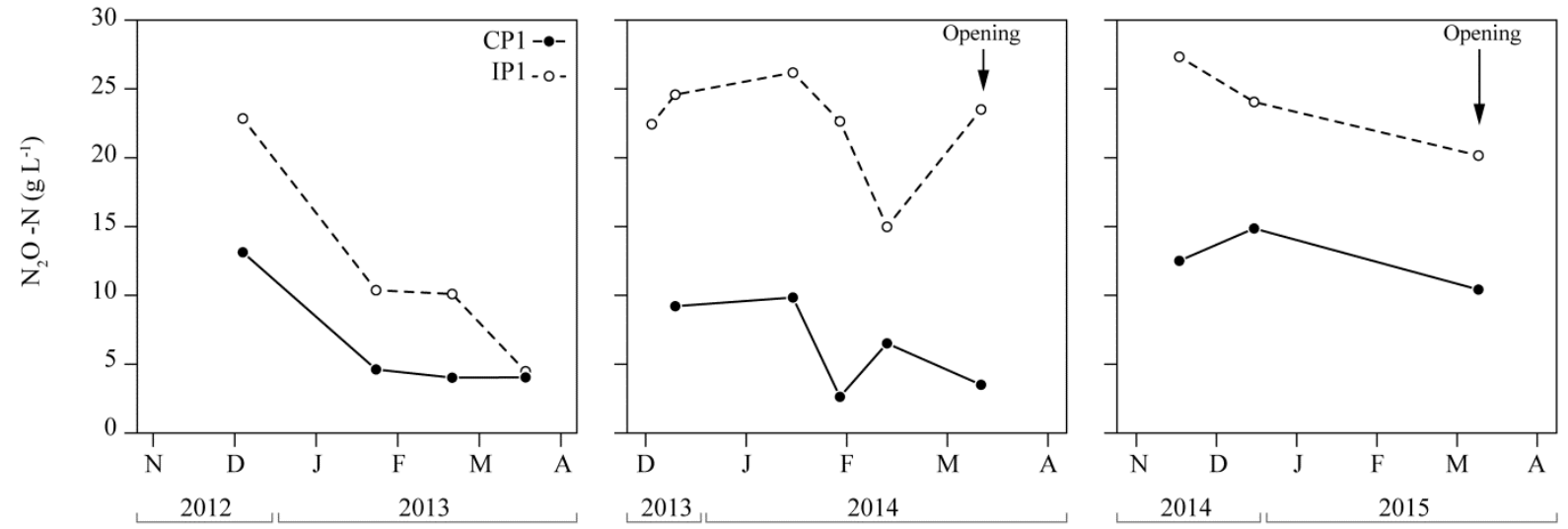

Figure S3. $\mathrm{N}_{2} \mathrm{O}-\mathrm{N}$ at CP1 and IP1 during Y0 (2012/13), Y1 (2013/14), and Y2 (2014/15).
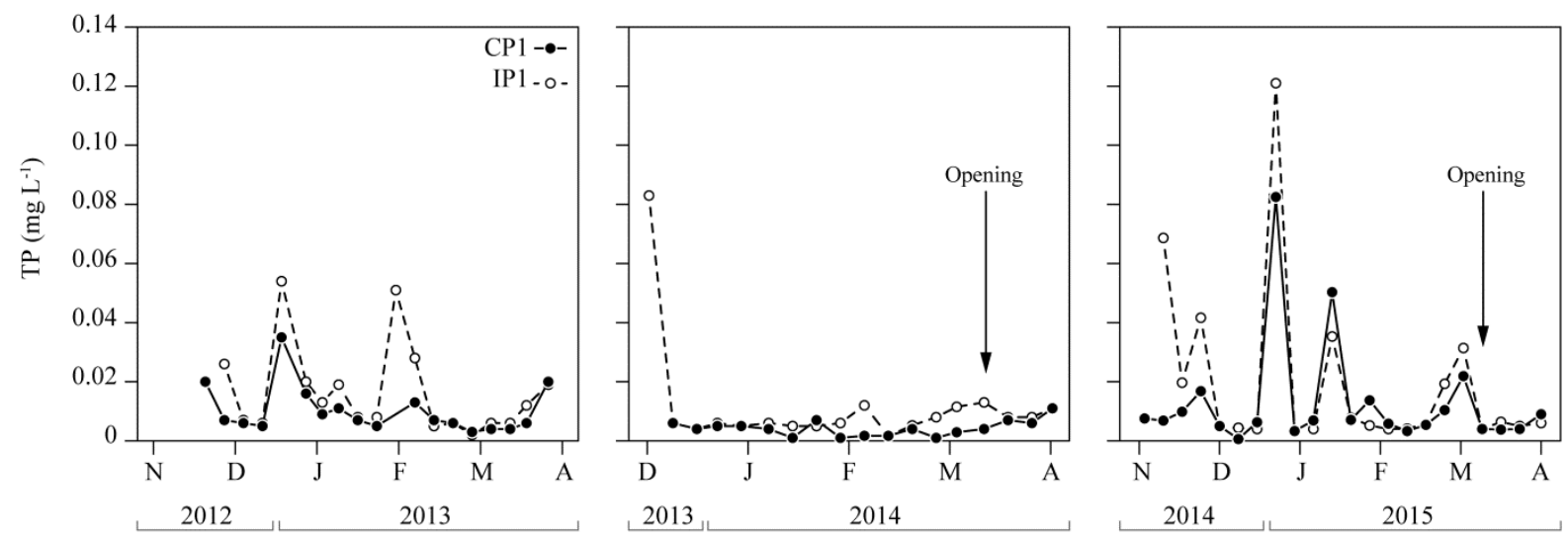

Figure S4. TP at CP1 and IP1 during Y0 (2012/13), Y1 (2013/14), and Y2 (2014/15). 

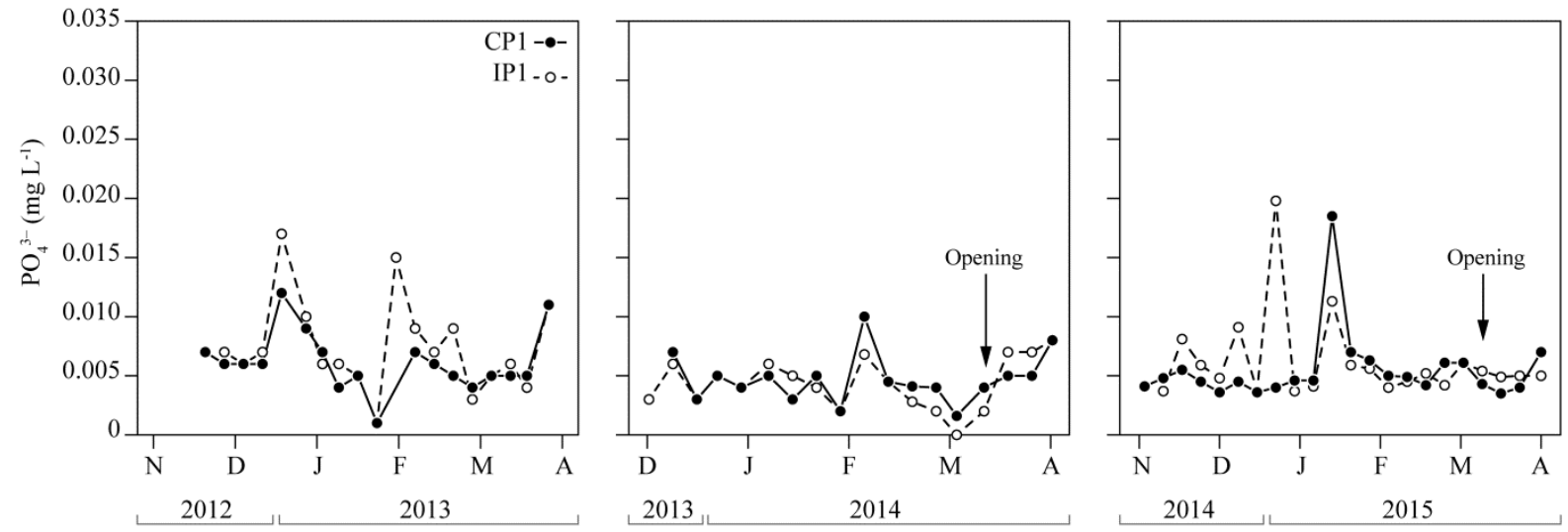

Figure S5. $\mathrm{PO}_{4}{ }^{3}$ - at CP1 and IP1 during Y0 (2012/13), Y1 (2013/14), and Y2 (2014/15).
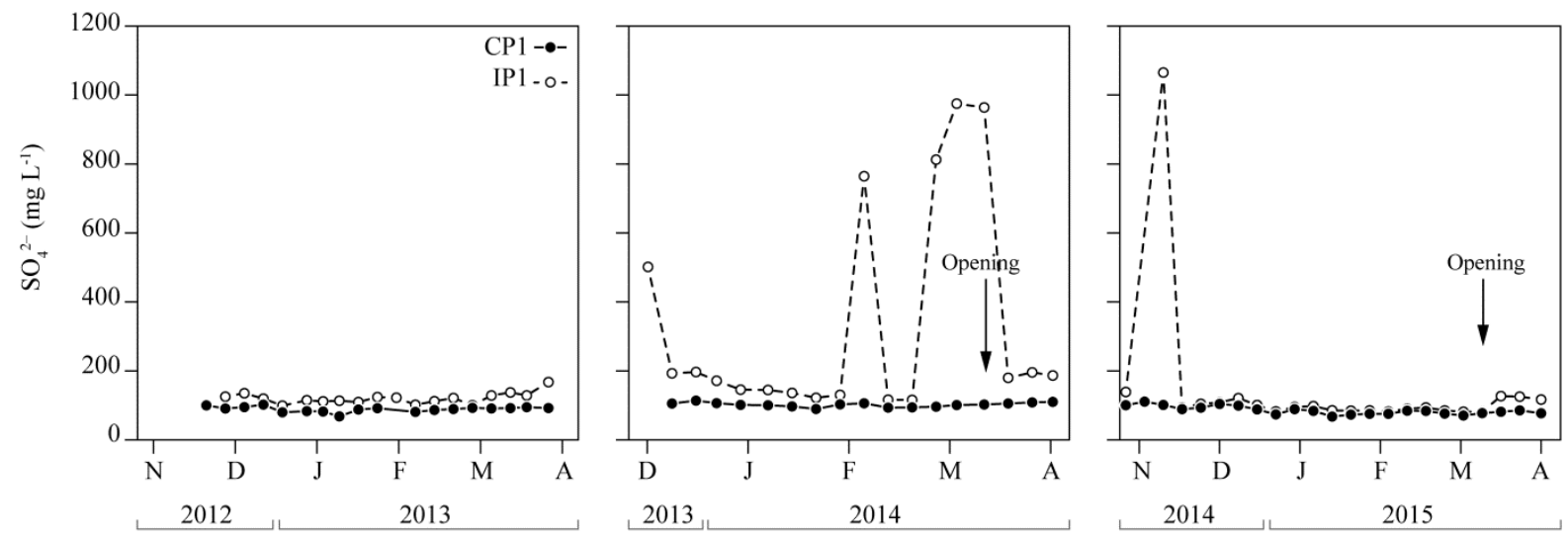

Figure S6. $\mathrm{SO}_{4}{ }^{2-}$ at CP1 and IP1 during Y0 (2012/13), Y1 (2013/14), and Y2 (2014/15).

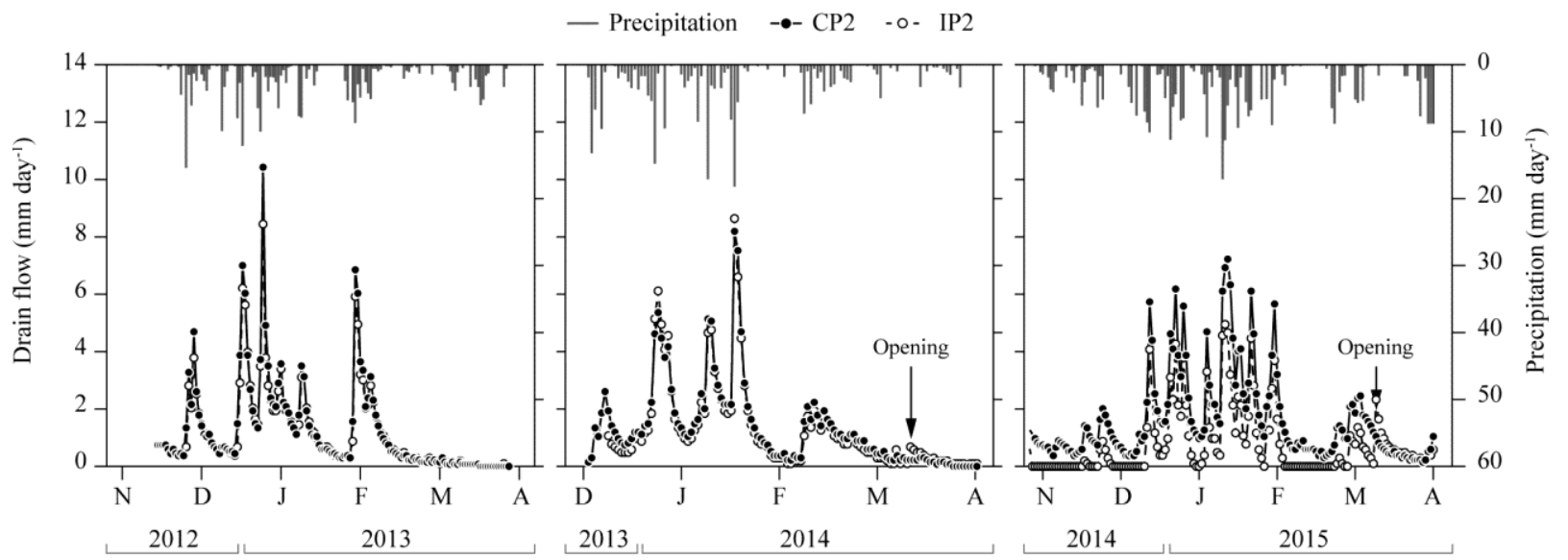

Figure S7. Drain flow and precipitation at CP2 and IP2 during Y0 (2012/13), Y1 (2013/14), and Y2 (2014/15). 

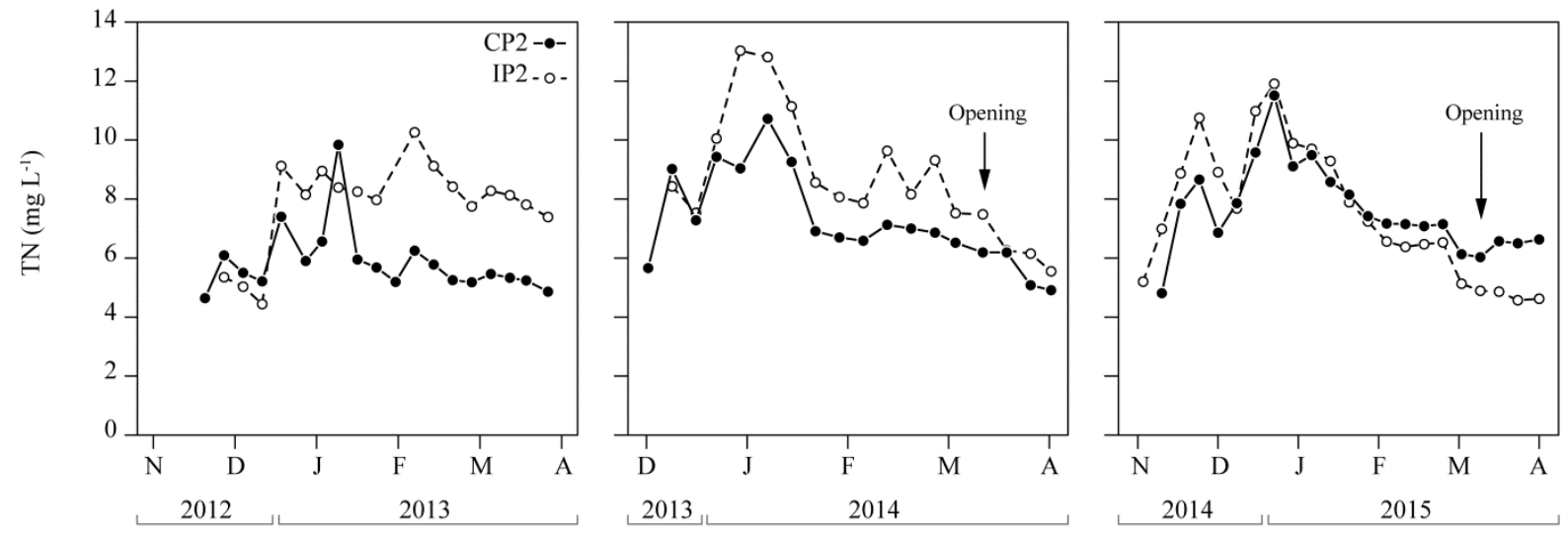

Figure S8. TN at CP2 and IP2 during Y0 (2012/13), Y1 (2013/14), and Y2 (2014/15).
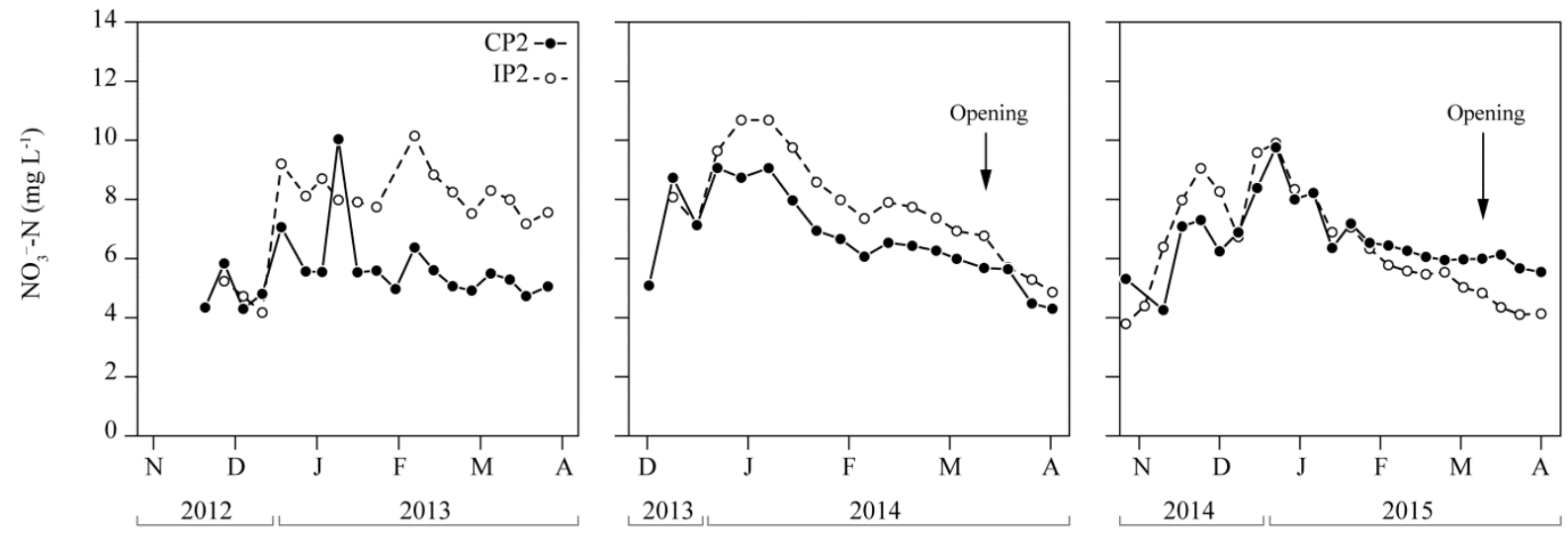

Figure S9. $\mathrm{NO}_{3}{ }^{-} \mathrm{N}$ at $\mathrm{CP} 2$ and IP2 during $\mathrm{Y} 0$ (2012/13), Y1 (2013/14), and Y2 (2014/15).
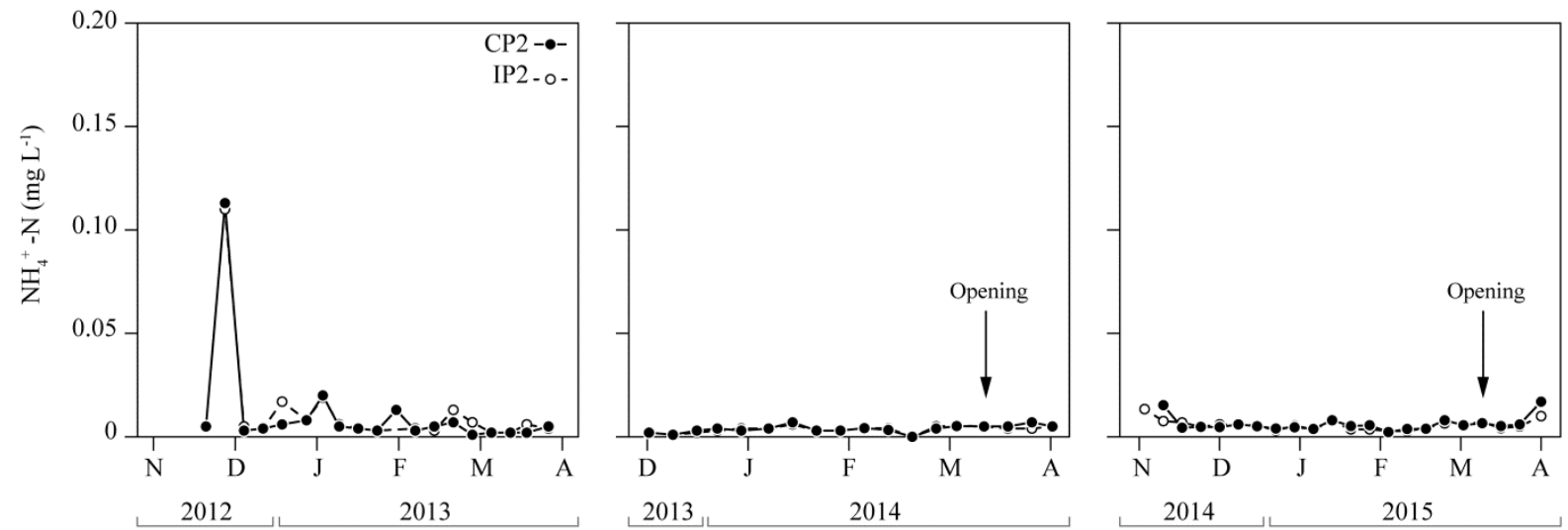

Figure S10. $\mathrm{NH}_{4}{ }^{+}$-N at CP2 and IP2 during Y0 (2012/13), Y1 (2013/14), and Y2 (2014/15). 

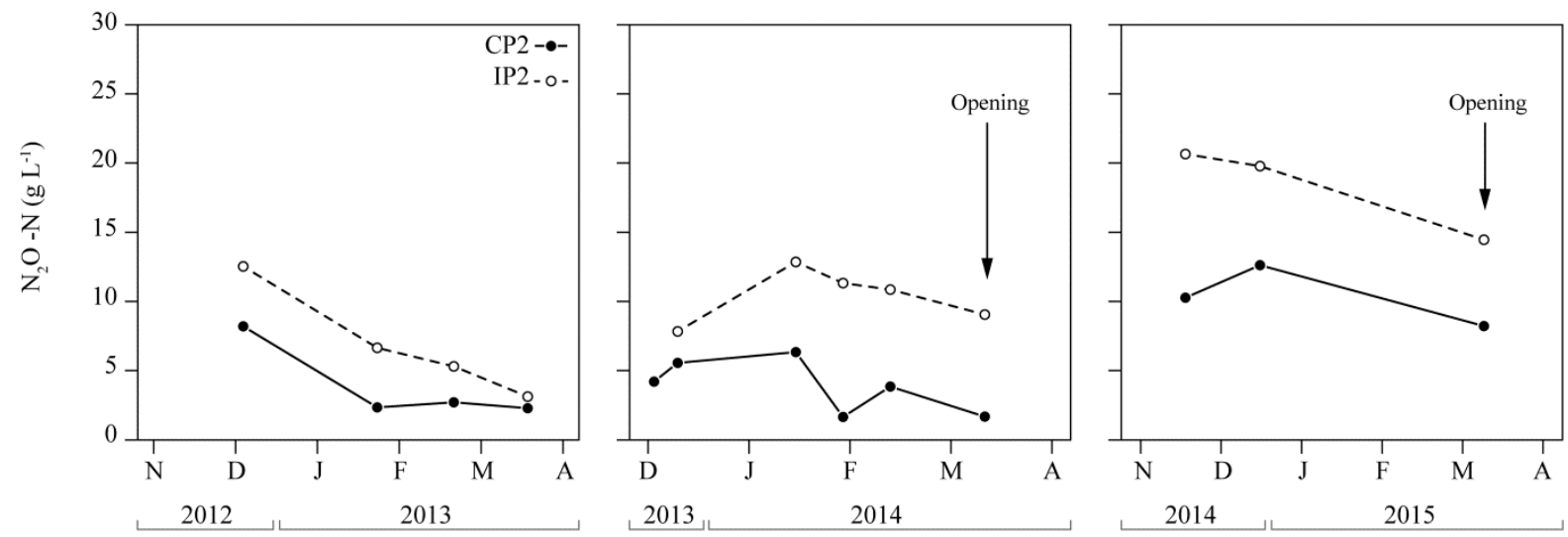

Figure S11. $\mathrm{N}_{2} \mathrm{O}-\mathrm{N}$ at CP2 and IP2 during Y0 (2012/13), Y1 (2013/14), and Y2 (2014/15).
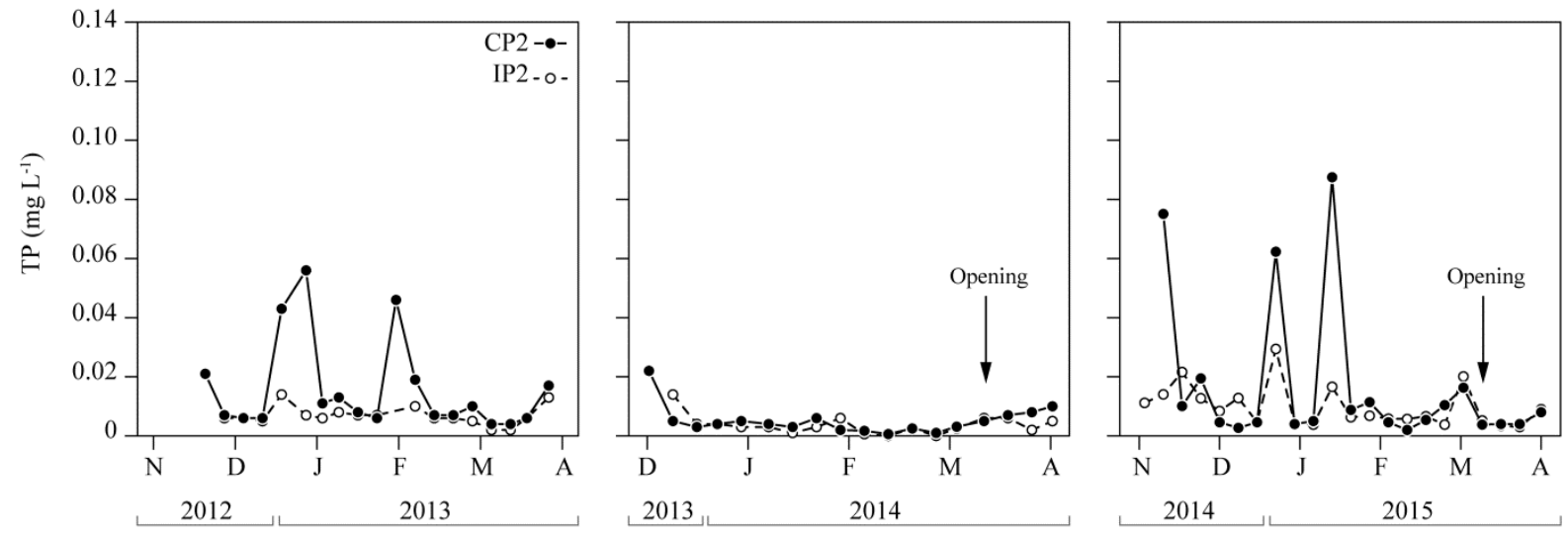

Figure S12. TP at CP2 and IP2 during Y0 (2012/13), Y1 (2013/14), and Y2 (2014/15).
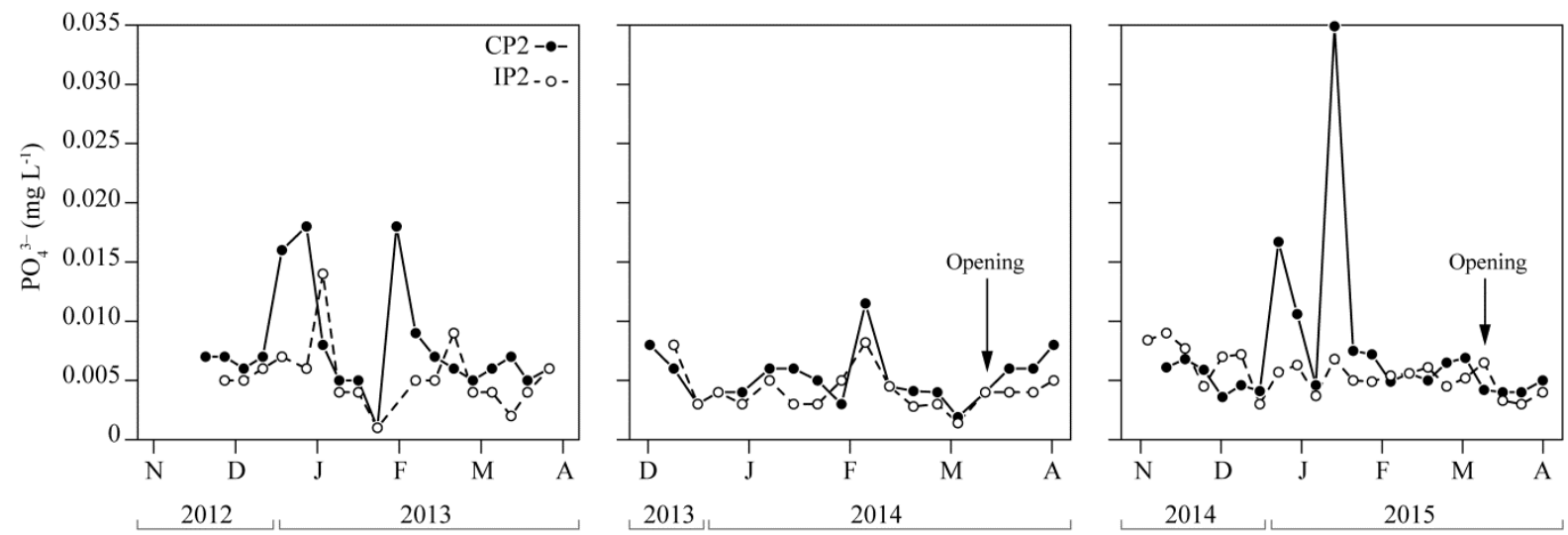

Figure S13. $\mathrm{PO}_{4}{ }^{3-}$ at CP2 and IP2 during Y0 (2012/13), Y1 (2013/14), and Y2 (2014/15). 


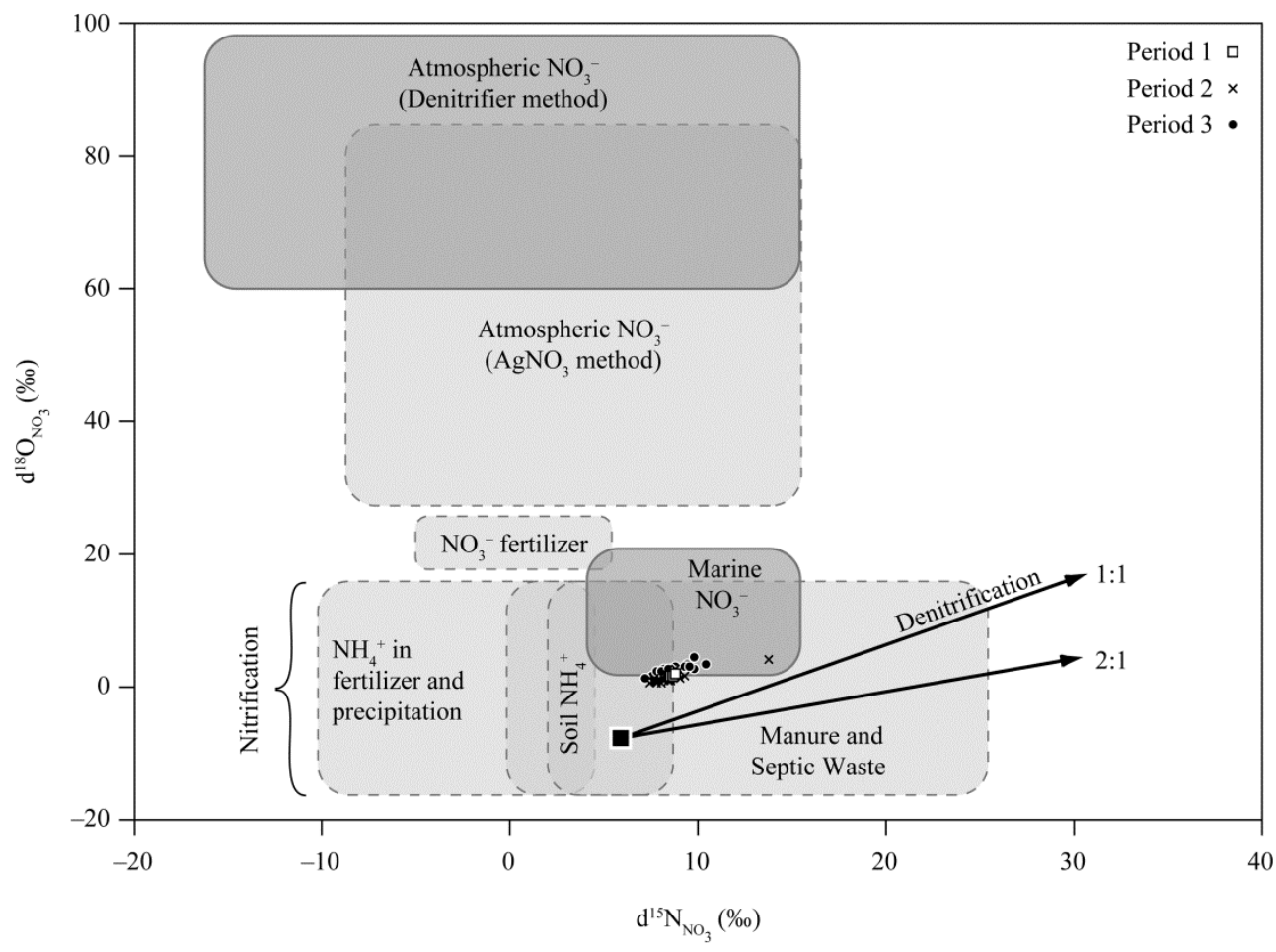

Figure S14. Measured ranges of $\delta^{15} \mathrm{~N}$ and $\delta^{18} \mathrm{O}$ of $\mathrm{NO}_{3}{ }^{-} \mathrm{N}$ from IP1, IP2, CP1, and CP2 in Y1 (2013/14) and Y2 (2014/15) plotted with typical ranges of $\delta^{15} \mathrm{~N}$ and $\delta^{18} \mathrm{O}$ from $\mathrm{NO}_{3}{ }^{-} \mathrm{N}$ sources adopted from Kendall et al. (2007).

\section{References}

Kendall, C., Elliott, E. M., and Wankel, S. D.: Tracing anthropogenic inputs of nitrogen to ecosystems, in: Stable Isotopes in Ecology and Environmental Science, 2nd edition ed., Blackwell Publishing, p. 375-449., 2007. 